\title{
Éditorial
}

\section{HISTORIQUE DU THERMALISME EN FRANCE}

\author{
Gilles-Pascal Husson
}

L'hydrologie thermale est la plus vieille des thérapeutiques médicales et parmi ces dernières c'est sans doute celle qui a subi le moins de changements.

Déjà, les Étrusques, apparus à la fin du VIIle siècle avant Jésus-Christ, font appel aux bienfaits des eaux et construisent, en Toscane, des bâtiments luxueux à proximité des sources et instaurent un système d'étude et de surveillance des eaux. En l'an 500 avant Jésus-Christ, Sophocle, Aristote, Plutarque et Aristophane chantaient les vertus des Eaux minérales. La construction d'établissements thermaux a été mise au jour en Égypte et en Grèce où l'émergence de l'eau, à cette époque, est assimilée à une divinité. L'eau jaillissant de la terre est considérée comme un phénomène surnaturel, éveillant des sentiments de vénération. Des prêtres et des prêtresses s'installent près des sources. Plusieurs stations célèbres de la Grèce antique existent encore de nos jours: Milo, Eubée, Cenchrées. Plus tard, les Romains adaptent et développent cet héritage thermal. Les installations romaines étaient grandioses et fort bien équipées avec des bains, des étuves, des salles de massages et de fumigations. De nombreux auteurs de l'époque, comme Horace, Juvenal, Galien,
Sénéque, Pline et même Cicéron citent le nom de ces stations: Apone, CEnaria, Baies et Abula, citée dans l'Éneïde, d'où les eaux étaient amenées jusqu'au palais de Néron. Les eaux sulfureuses d'Albula utilisées dans les affections nerveuses montrent clairement que les thérapeutes de l'époque ne se faisaient guère d'illusions sur l'état mental de leur empereur. Le dieu celte des sources Borvo, vénéré par les Gaulois devient Borvonus sous I'occupation romaine. Les Romains, sillonnant le monde, apportèrent leur pratique du thermalisme, améliorant les stations déjà existantes et en en créant d'autres. De nombreux vestiges, pièces de monnaies, objets, médailles, laissés dans presque toutes les stations, témoignent de cette activité. Les riches citoyens, certains malades, profitent de l'effet des eaux; De même les soldats de César, malades ou fatigués, peuvent ainsi se préparer à de nouvelles conquêtes. Certaines stations comme Aix-en-Provence, le Mont-Dore, Bagnères-de-Bigorre, Royat, Dax, Néris, Vichy accueillent encore maintenant de nombreux curistes.

Puis brusquement, au cours du IIIe siècle, les invasions surviennent et toutes ces villes florissantes s'écroulent. Les citoyens pensèrent à autre chose qu'au thermalisme et les Goths achevèrent 
au Ve siècle de démolir ce qui restait encore debout.

Le Haut-Moyen Age ne voit pas la pratique thermale avec un grand enthousiasme. C'est Charlemagne qui insuffle un certain renouveau.

Le retour des Croisades, au XIII siècle, provoque à nouveau des maladies contractées dans des pays lointains qui peuvent être soignées dans les thermes.

Avec la Renaissance apparaît un thermalisme de cour. Rabelais, Montaigne et également Henri II et Henri III vantent les bienfaits des eaux thermales.

C'est Henri IV, en 1605, qui inaugure le premier système d'inspection des établissements thermaux et la législation sur les eaux minérales. C'est au premier médecin du Roi, un certain La Rivière, qu'est confié le poste de surintendant général des bains et fontaines minérales du Royaume. Ce dernier nomme dans chaque province des intendants des eaux (médecins) qui l'aideront dans sa tache de "surveillance, d'étude et de maîtrise d'œuvre".

C'est grâce à la littérature française (lettres de la marquise de Sévigné, œuvre de Boileau) que I'on peut imaginer ce que représente le thermalisme d'alors.

En 1666 commence certains travaux sur la crédibilité des eaux minérales: I'Académie de Médecine réalise un premier recensement général des eaux minérales françaises. Le 25 avril 1772 est créée la commission Royale de Médecine qui comprend des inspecteurs généraux des eaux minérales et qui ont pour mission de contrôler et de délivrer les permis de d'exploitation pour la commercialisation des eaux.

La Révolution, dans son grand chambardement, supprime tout cela. Des "médecins inspecteurs des eaux minérales de France " sont créés et le contrôle des eaux et leur exploitation sont transférés aux autorités locales (cantons et départements).

Un arrêté du Directoire stipule que "les eaux minérales étant un don de la nature, elles appartiennent à tous et font partie des ressources publiques" Le premier et le Second Empire contribuent au développement du thermalisme. MarieAmélie épouse de Louis Philippe ainsi que l'impératrice Eugénie donnent leur nom à des stations. Napoléon III, par ses nombreux séjours dans les stations, entre autres à Vichy, les fait connaître aux Français.
En 1820, l'Académie Royale de Médecine remplace l'ancienne Société Royale. Elle supervise toutes les formalités d'ouverture de nouvelles sources ainsi que les permis d'exploitation. C'est en 1848 qu'apparaît un décret instituant un périmètre de protection de mille mètres de rayon où les forages sont interdits de façon à lutter contre le pillage des sources.

La première guerre mondiale suivie de la crise financière de 1929 et même la deuxième guerre mondiale marquent une longue période de baisse de la fréquentation des stations thermales. Un grand principe de reconnaissance des stations hydrominérales attachées aux communes sur lesquelles elles sont situées géographiquement est posé dans la loi du 24 septembre 1919.

Un nouveau concept de thermalisme social voit le jour vers le début des années quarante avec l'envoi en cure proposé à toute personne en ayant médicalement besoin. Des circulaires ministérielles en 1947 permettent la prise en charge pour certains soins thermaux de la totalité ou d'une partie des dépenses. Depuis cette date le sort du thermalisme reste étroitement lié à la politique gouvernementale et à celle de la Sécurité Sociale. En 1980, Guy Ebrard, auteur d'un rapport demandé par le président de la République écrivait: "L'invention de la Sécurité Sociale a eu trois conséquences majeurs sur le thermalisme français : 1) Les assurés sociaux ont progressivement constitué l'essentiel de la clientèle des stations, en se substituant à la clientèle de jadis. 2) Les cures thermales ont revêtu un caractère médical de plus en plus accentué. 3) Ces cures thermales sont prescrites à plus de $90 \%$ par des médecins et effectués pratiquement à $100 \%$ sous contrôle médical "

La volonté de conserver le patrimoine français provoque d'importantes modifications et de constructions nouvelles d'établissements, mieux adaptés aux progrès de la recherche.

L'histoire thermale apparaît ainsi comme une succession d'efforts importants, grâce auxquels la médecine thermale a su imposer sa spécificité, malgré des périodes, parfois longues, d'indifférences ou de relâchement.

Le nombre de curistes est en progression lente depuis la fin de la deuxième guerre mondiale avec certaines fluctuations en rapport notamment avec des mesures restrictives prises à partir des années cinquante. Actuellement la France compte plus d'un millier de sources et une centaine de stations agrées par la Sécurité Sociale. 


\section{Statistiques de frequentation des Ctablissements thermaux. Annce 1995}

(Syndicat National des Etablissements Thermaux 10 rue de la Trémoille 75008 Paris)

\section{ÉTABLISSEMENTS}

Aix-les-Bains

Aix-Marlioz

Aix-en-Provence

Alet-les-Bains

Allevard-les-Bains

Amélie-les-Bains

Amneville

Argelès-Gazost

Aulus-les-Bains

Aurensan

Avène-les Bains

Ax-les-Thermes

Bagnères-de-Bigorre

Bagnoles-de-L'Orne

Bagnols-les-Bains

Bains-les-Bains

Balaruc

Barbazan

Barbotan-les-Thermes

Barèges-Sers

Beaucens

Berthemont

Boulou (Le)

Bourbon-Lancy

Bourbon-l'Archambault

Bourbonne

Bourboule (La)

Brides-les-Bains

Cambo-les-Bains

Camoins-les-Bains

Capvern-les-Bains

Castera-Verduzan

Cauterets

La Chaldette

Challes-les-Eaux

Châteauneuf-les-Bains

1356

269

192

1165

8237

7944

13479

1546

3083

34060

291

16825

2856

761

1033

1622

3407

4659

14066

17700

12084

6257

3744

5137

205

9398

50

3828

603
40116

Châtel-Guyon

11000

Chaudes-Aigues

2217

Claos

545

Contrexeville

1010

Cransac

2341

Dax

Digne-les-Bains

53534

8311

Divonne

3453

Les Eaux-Bonnes

1170

1962

1390

3583

1992

1619
Enghien-les-Bains

Eugénie-les-Bains

Evaux-les-Bains

Evian-les-Bains

Forges-les-Eaux

Les Fumades

Gréoux-les-bains

Guagno-les-Bains

Jonzac

Lamalou-les-Bains

La Léchère

Lons-le-Saunier

Luchon

Luxeuil-les-Bains

Luz-Saint-Sauveur

Maizières

Moltig-les-Bains

Montbrun

Le Mont-Dore

Montrond-les-Bains

Morsbronn

Néris-les-Bains

Neyrac-les-Bains

Niederbronn-les-bains

Pechelbronn

Pietrapola

Plombières

Préchacq-les-Bains

La Preste

Propiac

Rennes-les-Bains

Rochefort

La Roche-Posay

\section{$F$}

2727

23784

251

5039

5049

8989

2308

22746

1560

1630

1330

514

10770

2035

6167

6520

1624

3993

306

4632

1496

3023

1373

9655

8978 
Royat

Sail-les-Bains

Saint-Amand

Saint-Christau

Saint-Gervais

Saint-Honoré

Saint-Lary

Saint-Laurent

Saint-Nectaire

Saint-Paul-les-Dax

Salies-de-Béarn

Salies-du-Salat

Salins-les-Bains

Santenay

16052
$F$
$F$
529
4395
4397
2216
1253
1117
11809
2670
1778
1275
$F$

Saubusse

Saujon

Tercy-les-Bains

Thonon-les-Bains

Uriage

Ussat-les-bains

Vals-les-Bains

Vernet-les-Bains

Vichy

Vittel

Zigliara

TOTAL

F: fermé
1480

1450

2078

738

7610

$$
\mathrm{F}
$$

2992

3982

12795

4135

146

588590
Comme ce précédent tableau le démontre, le thermalisme est toujours bien vivant. Certes des évolutions et des adaptations au temps présent et au $X X \mathrm{I}^{\mathrm{e}}$ siècle tout proche sont nécessaires et en cours de réalisation d'ailleurs.

Les acteurs du thermalisme voient, entre autres, quatre grands thèmes à approfondir : 1) Les définitions des critères de qualité de la ressource thermale en eau, avec la protection de cette ressource comme patrimoine naturel. 2) Les problèmes de quantités d'eau sous-tirées du sous-sol et de la capacité d'accueil des établissements. 3) Les mêmes garanties d'hygiène pour chaque curiste dans tous les établissements thermaux. 4) Un regard sur la façon dont fonctionne le thermalisme dans différents pays européens, notamment en Allemagne où les établissements thermaux participent également à certains séjours des convalescents.

Reprenant l'idée du début de cet éditorial sur la plus vieille des thérapeutiques médicales, on ne peut être que convaincu qu'il reste encore au thermalisme de nombreuses années à demeurer présent au côté des autres pratiques modernes et futures.

Le rédacteur en chef. 